\title{
The need for culture swabs in laparoscopically treated appendicitis
}

\author{
Jakub Kenig, Piotr Richter \\ $3^{\text {rd }}$ Department of General Surgery, Jagiellonian University Medical College, Krakow, Poland
}

Videosurgery Miniinv 2013; 8 (4): 310-314

DOI: 10.5114/wiitm.2011.35002

\begin{abstract}
Introduction: Appendicitis remains the most common cause of an acute abdomen. Obtaining intra-abdominal cultures is routine surgical practice. There are studies showing no efficacy of such procedures in cases where open appendectomies are performed.

Aim: The goal of this study was to assess the need for obtaining intra-abdominal cultures during laparoscopic appendectomies.

Material and methods: Between 2007 and 2012, 369 patients were operated on with the diagnosis of histopathologically proven acute appendicitis. Sixty-two percent of them were operated on using laparoscopic techniques. The microbiological assessment was routinely done for the open procedures and in $42 \%$ of cases that underwent a laparoscopic operation.

Results: In 57\% (134) the swabbing results were negative. Among 43\% (102) of the patients with a positive result, Escherichia coli was isolated in 76.5\% (78), Proteus mirabilis in 13.7\% (14), Pseudomonas aeruginosa in 4.9\% (5) and Citrobacter freundii in $4.9 \%$ (5). Five cases had bacteria resistant to the antibiotic given preoperatively (that is $4.9 \%$ of all positive cultures and $1.4 \%$ of all operated patients). However, these cases did not affect the incidence of postoperative complications. Consideration of the postoperative morbidity showed that there was no statistically significant difference between the laparoscopic group with and without intra-operative swabbing $(p>0.05)$.

Conclusions: The postoperative patient outcome was more dependent on the pathology of the appendix than on the results of the microbiological assessment at the time of surgery. Hence, routine intra-operative cultures during laparoscopic appendectomies appear to have little value in patient management. Swabbing during laparoscopic procedures should be limited to only selected high-risk groups.
\end{abstract}

Key words: culture swabs, laparoscopic appendectomy, appendicitis.

\section{Introduction}

Appendicitis remains the most common cause of an acute abdomen. Preoperative administration of broad-spectrum antibiotics and obtaining intraabdominal cultures are routine surgical practice. However, with the increased use of laparoscopic techniques, the standard method for treatment of appendicitis with routine microbiological evaluation of the intra- abdominal fluid has significantly decreased. This might be due to the results of studies showing no efficacy of such procedures in cases where open appendectomies are performed. The sampling of intra-abdominal fluid is more difficult with laparoscopic surgery; special suction devices are needed for the fluid sampling. The results of a meta-analysis of 56 studies compared laparoscopic to open appendec- 
tomies in adult patients and showed that although wound infections were less likely following a laparoscopic appendectomy, the cases that underwent a laparoscopic procedure had an increased prevalence of intra-abdominal abscess formation [1]. One possible reason could be the lack of microbiological assessment in the laparoscopic group, which in turn could result in inappropriate treatment.

\section{Aim}

The goal of this study was to assess the need for obtaining intra-abdominal cultures during laparoscopic appendectomies.

\section{Material and methods}

Between 2007 and 2012, 369 patients (47\% female, 53\% male) were operated on at the Third Department of Surgery, Jagiellonian University Medical College, with the diagnosis of histopathologically proven acute appendicitis. The mean age was 35.8 (range: 16-92 years). Sixty-two percent (229) of them were operated on using laparoscopic techniques. Based on the histopathology report, 13\% (48) of the cases were diagnosed as simple acute appendicitis, $55 \%$ (203) as phlegmonous, 23\% (85) gangrenous, and $9 \%$ (33) were diagnosed as necrotic appendicitis with perforation.

All patient histories were reviewed and their gender, age, type of operation, and histology reports were recorded to confirm the diagnosis and determine the histopathological subtype of appendicitis; microbiological reports and the occurrence of any postoperative complications during the hospitalization and follow-up in the outpatient clinic were reviewed.

\section{Surgical procedure}

The patients were divided into three groups based on the technique used for the operation: open procedure (all patients had microbiological reports), a laparoscopic group with and one without intraabdominal microbiological assessment. All patients received a single preoperative intravenous infusion of an antibiotic (amoxicillin clavulanate), which was continued up to the seventh postoperative day. In cases with penicillin allergy, this agent was generally substituted with ciprofloxacin.

\section{Microbiology}

Each intra-operative microbiological swab was placed in transport medium. All specimens were sent to the laboratory immediately after collection, cultured on site in the same laboratory, then bacterial strains were isolated, and their sensitivities to antibiotics identified.

\section{Histopathology}

The appendicitis was classified based on the histopathological report as a non-inflamed appendix, pyogenic acute appendicitis (neutrophil infiltration), necrotizing appendicitis (if necrosis of the appendicular wall occurred) or necrotizing complicated by perforation (microscopic evidence of perforation of the wall).

\section{Statistical analysis}

Qualitative and quantitative data are used to describe the study results. Quantitative parameters are expressed as the mean value \pm standard deviation. The remaining cases were coded using Arabic numerals. The data were analyzed using the Statistica 10.0 software suite (StatSoft). The Shapiro-Wilk W and the Kolmogorov-Smirnov tests with the Lilliefors correction were used to verify the normality of the distribution of the results. Based on the normality of the distribution, the data were analyzed using parametric or non-parametric tests. The null hypothesis (H0) was rejected at the established level of $\alpha=0.05$.

\section{Results}

The microbiological assessment was routinely done for the open procedures. In the patients who underwent a laparoscopic operation, microbiological assessment was performed in $42 \%$ (96) of cases. In $57 \%$ (134) the swabbing results were negative. Among 43\% (102) of the patients with a positive result, Escherichia coli was isolated in $76.5 \%$ (78), Proteus mirabilis in $13.7 \%$ (14), Pseudomonas aeruginosa in 4.9\% (5) and Citrobacter freundii in 4.9\% (5). Five cases had bacteria resistant to the antibiotic given preoperatively, i.e. $4.9 \%$ of all positive cultures and $1.4 \%$ of all operated patients. The proportion of patients with a positive culture increased with the severity of the histopathological diagnosis (Table I). Bacteria resistant to the antibiotics given preopera- 
Table I. The proportion of patients with a positive culture with bacteria resistant to the preoperative antibiotic, grouped by histopathological subtype of appendicitis

\begin{tabular}{|lcc|}
\hline $\begin{array}{l}\text { Histopathological } \\
\text { subtype }\end{array}$ & Percentage of positive cultures & $\begin{array}{c}\text { Percentage of bacteria resistant to the } \\
\text { preoperative antibiotic }\end{array}$ \\
\hline Simple acute appendicitis & $16.7 \%(8)$ & $0 \%(0)$ \\
\hline Phlegmonous appendicitis & $20.2 \%(41)$ & $0 \%(0)$ \\
\hline Gangrenous appendicitis & $38.8 \%(33)$ & $6 \%(2)$ \\
\hline Gangrenous with perforation & $60.6 \%(20)$ & $15 \%(3)$ \\
\hline
\end{tabular}

Table II. The proportion of patients that underwent a laparoscopic procedure, with positive cultures and resistant bacteria to the preoperative antibiotic, grouped by histopathological subtype of appendicitis

\begin{tabular}{|lcc|}
\hline $\begin{array}{l}\text { Histopathological } \\
\text { subtype }\end{array}$ & $\begin{array}{c}\text { Percentage of positive } \\
\text { cultures }\end{array}$ & $\begin{array}{c}\text { Percentage resistant bacteria } \\
\text { of to the preoperative antibiotic }\end{array}$ \\
\hline Simple acute appendicitis & $8.6 \%(3)$ & $0 \%(0)$ \\
\hline Phlegmonose appendicitis & $15.7 \%(18)$ & $0 \%(0)$ \\
\hline Gangrenous appendicitis & $20 \%(12)$ & $17 \%(2)$ \\
\hline Gangrenous with perforation & $42.1 \%(8)$ & $0 \%(0)$ \\
\hline
\end{tabular}

tively were cultured only in 2 and 3 patients with gangrenous and gangrenous with perforation appendicitis, respectively. However, these cases did not affect the incidence of postoperative complications. Among this group, 4 patients had no postoperative complications and 1 patient had a superficial wound infection.

Taking into consideration the patient age, 70\% (31) of elderly patients (age $>65$ years old) had a positive culture compared to $22 \%$ (71) of younger patients; this was a statistically significant difference. The mean length of hospitalization was $4.5 \pm 3.2$ days (range: $2-28$ ) in the open group and $3.2 \pm 1.3$ days (range: 2-12) in the laparoscopic group, including $80 \%$ of patients discharged on the second postoperative day.

Comparison of the open and laparoscopic procedures showed that among $43.4 \%$ (61) and $42.7 \%$ (41) of patients who had swabbing, bacteria were isolated $(p>0.05)$. Table II shows the proportion of patients who underwent the laparoscopic procedure, with a positive culture that had bacteria resistant to the preoperative antibiotic, grouped by histopathological subtype of appendicitis.

Consideration of the postoperative morbidity showed that there was no statistically significant difference between the laparoscopic group with and without intra-operative swabbing $(p>0.05)$. In 2 patients bacteria resistant to the preoperative antibiotic were isolated. However, the antibiotic regimen was not changed due to the good postoperative course of the patients.

\section{Discussion}

The main aim of intra-operative swabbing is to identify cases with pathogens resistant to administered antibiotics. However, the results of this study show that this number was very small (4.9\% of all positive cultures and only $1.4 \%$ of all operated patients). In addition, there was no statistically significant difference noted when postoperative morbidity (4 patients had no postoperative complications, 1 patient had superficial wound infection in this group) was compared to the group sensitive to the preoperatively administered antibiotics. Because of the very good postoperative course (no clinical, biochemical or imaging signs of infectious complications) among all patients, the antibiotic regimen was not changed. Furthermore, the average time for obtaining the results of culture and sensitivity at our institution was between 2 and 3 days. Most of the patients treated 
laparoscopically had already been discharged from the hospital by that time. These findings are consistent with prior reports. Gladman et al. found that $2 \%$ (11 of 463 patients) of the organisms identified were resistant to broad-spectrum antibiotics. Foo et al. reported isolation of $4.1 \%$ of resistant organisms and in the group presented by Moawad et al. only $1 \mathrm{pa}$ tient $(0.85 \%)$ with resistance was found. The authors also concluded that neither the presence of a positive intra-operative culture, nor the isolation of resistant organisms was significant in predicting complications with infections [2-4]. Moreover, other authors have also reported no change or only $7-16 \%$ of patients had changes in their antibiotic therapy based on swab results [3, 5-9].

In this study swabs were taken in $64 \%$ of the operations; $43 \%$ of them yielded positive cultures. Other authors present similar results, with wide variation in the positive culture rates, ranging from $18 \%$ to $95 \%$ [3, 9-12]. As far as laparoscopic procedures are concerned, positive cultures were noted in $42.7 \%$ of patients. There was no statistically significant difference in the postoperative course among patients after laparoscopy with and without cultures taken. In addition, there was no statistically significant difference in the incidence of intraperitoneal abscess formation between the open group and the laparoscopic group with and without microbiological assessment. These findings are consistent with the meta-analysis reported by Sauerland et al. [1]. These results suggest that the predicted bacteria that infect the acute abdomen in patients with appendicitis are effectively treated by current antibiotic regimens. Only one study found a significant difference between the open and laparoscopic group and this might be explained by the difference in the size of the study groups (88 patients in the open and only 23 in the laparoscopic group). Most prior studies assessed the entire group together (open and laparoscopic), not showing the data for the two groups separately [14].

The results of this study showed an increase in positive cultures with an increase in the age of the patients, $70 \%$ of elderly patients (age $>65$ years old) in comparison to only $22 \%$ in younger patients; this is consistent with prior results. Therefore, the data suggest that swabbing during laparoscopic procedures should be performed in only selected high-risk groups, including cases with necrotic and complicated appendicitis and elderly patients [9-12, 15, 16].
This study was limited by its retrospective design and the absence of randomization. However, the antibiotic regimen, surgical procedure, swab collection and assessment were all standard procedures.

\section{Conclusions}

The postoperative patient outcome was more dependent on the pathology of the appendix than on the results of the microbiological assessment at the time of surgery. Hence, routine intra-operative cultures during laparoscopic appendectomies appear to have little value in patient management. Swabbing during laparoscopic procedures should be limited to only selected high-risk groups, including cases with necrotic and complicated appendicitis and elderly patients.

\section{References}

1. Sauerland S, Jaschinski T, Neugebauer EA, et al. Laparoscopic versus open surgery for suspected appendicitis. Cochrane Database Syst Rev 2010; 6: CD001546.

2. Gladman MA, Knowles CH, Gladman LJ, et al. Intra-operative culture in appendicitis: traditional practice challenged. Ann R Coll Surg Engl 2004; 86: 196-201.

3. Foo FJ, Beckingham IJ, Ahmed I. Intra-operative culture swabs in acute appendicitis: a wste of recources. Surgeon 2008; 6: 278-81.

4. Moawad M, Dasmohapatra S, Justin T, et al. Value of intraoperative abdominal cavity culture in appendicectomy: a retrospective study. J Clin Pract 2006; 60: 1588-90.

5. Kwiatkowski A, Paśnik K, Trojanowski P. Late caecal fistula after laparoscopic appendectomy managed mini-invasively: case report. Videosurgery Miniinv 2011; 6: 246-8.

6. Gołębiewski A, Łosin M, Murawski M, et al. One, two or three port appendectomy; a rational approach. Videosurgery Miniinv 2013 (e-pub, ahead of print) manuscript accepted for publication.

7. Fraulin FO, Thurston OG. Value of cultures of tissue samples taken at operation for lower intestinal perforation. Can J Surg 1993; 36: 261-5.

8. Jaffers GJ, Pollock TW. Intraoperative culturing during surgery for acute appendicitis. Arch Surg 1981; 116: 866-8.

9. Mosdell DM, Morris DM, Fry DE. Peritoneal cultures and antibiotic therapy in pediatric perforated appendicitis. Am J Surg 1994; 167: 313-6.

10. Seco JL, Ojeda E, Reguilon C, et al. Combined topical and systemic antibiotic prophylaxis in acute appendicitis. Am J Surg 1990; 159: 226-30.

11. Henderson J, Forshaw MJ, Power DM, et al. Should surgeons swab during appendicectomy? An audit. J R Coll Surg Edinb 2001; 46: 367-9.

12. Pal KM, Khan A. J. Appendicitis: a continuing challenge. Pak Med Assoc 1998; 48: 189-92.

13. Kiudelis M, Ignatavicius P, Zviniene K, et al. Analysis of intracorporeal knotting with invaginating suture versus endoloops in appendiceal stump closure. Videosurgery Miniinv 2013; 8: 69-73. 
14. Power DM, Foreshaw MJ, Henderson J, et al. A review of emergency appendicectomy: should surgeons swab? The Internet Journal of Surgery 2002; 3 (2).

15. Kokoska ER, Silen ML, Tracy Jr TF, et al. The impact of intraoperative culture on treatment and outcome in children with perforated appendicitis. J Pediatr Surg 1999; 34: 749-53.

16. Duda M, Gryga A, Czudek S, et al. Twenty years of minimally invasive surgery in the Czech Republic. Videosurgery Miniinv 2011; 6: 42-7.

Received: 28.02.2013, accepted: 24.03.2013. 